\title{
Microincision Vitrectomy Trocars - Redefining Surgical Practices Through a New Range of Applications
}

\author{
João Pedro Marques, ${ }^{1}$ Cátia Azenha ${ }^{1}$ and João Figueira'1,2 \\ 1. Ophthalmologist, Centro Hospitalar e Universitário de Coimbra (CHUC), Coimbra, Portugal; \\ 2. Professor of Ophthalmology, Faculty of Medicine of the University of Coimbra (FMUC), Coimbra, Portugal
}

\begin{abstract}
Transconjunctival microincision vitrectomy surgery (MIVS) has grown increasingly popular among vitreoretinal surgeons over the last few years. Technical advances have led to the development of cutting-edge vitrectomy systems and instruments that significantly contributed to the success of MIVS. Trocar evolution has added extra safeness and effectiveness to the technique. In the hands of an experienced surgeon, microincision vitrectomy trocars offer a new range of applications that can redefine surgical practices and facilitate otherwise complex surgical techniques.
\end{abstract}

\section{Keywords}

Small-gauge vitrectomy, surgical instruments, trocars, surgical procedures, incision, sutureless, new techniques

\begin{abstract}
Disclosure: João Pedro Marques and Cátia Azenha have no conflicts of interest to declare. João Figueira has been a consultant for Alimera, Allergan, Bayer, Novartis and THEA. No funding was received in the publication of this article.

Open Access: This article is published under the Creative Commons Attribution Noncommercial License, which permits any non-commercial use, distribution, adaptation and reproduction provided the original author(s) and source are given appropriate credit.

Received: 22 April 2015 Accepted: 22 June 2015 Citation: European Ophthalmic Review, 2015;9(1):56-8

Correspondence: João Figueira, Department of Ophthalmology, Centro Hospitalar e Universitário de Coimbra Praceta Mota Pinto, 3000-075 Coimbra, Portugal.

E: joaofigueira@oftalmologia.co.pt
\end{abstract}

\section{Introduction}

Since the introduction of pars plana vitrectomy by Robert Machemer in 1970, technical advances have led to the development of progressively more capable and proficient commercial vitrectomy systems. Small-gauge vitrectomy became widely available in 2004 and has grown increasingly popular among vitreoretinal surgeons., ${ }^{1,2}$ Transconjunctival microincision vitrectomy surgery (MIVS) with 27-, 25- or 23-gauge instrumentation has shown several advantages over the traditional 20-gauge surgery. ${ }^{3}$ These include sutureless incisions, faster wound healing, less conjunctival scarring, decreased operating time, less surgically induced astigmatism, improved patient comfort and less post-operative inflammation with early visual recovery. ${ }^{2-6}$

Critical concerns accompanying the use of and expanding indications for 23- and 25-gauge MIVS and wound sealing-related complications (hypotony, choroidal detachment and post-operative infections) have been addressed, ${ }^{2,3}$ making it common practice among some vitreoretinal surgeons to close the entry wounds of 23- and even 25-gauge MIVS with resorbable transconjunctival scleral sutures. These concerns are less evident with 27-gauge systems, even though further refinement of the 27-gauge instrument functionality and rigidity are critical to allow the widespread use of this system for the whole spectrum of vitreoretinal diseases. ${ }^{3}$

Trocar evolution has accompanied the advances in commercial vitrectomes and significantly contributed to the safeness and effectiveness of today's MIVS. Differences in blade design and changing the insertion technique from 1- to 2-step entry ${ }^{5}$ have modified the incision architecture, thus allowing sutureless procedures. By providing a closed system, valved cannulas eliminate the need for plugs and decrease instrument exchanges, hence contributing to a better control of intraocular pressure and eye outflow intraoperatively. Furthermore, in the hands of experienced surgeons, microincision vitrectomy trocars offer a new range of applications that can redefine surgical practices and facilitate otherwise complex surgical techniques.

We aim to describe our personal experience with this invaluable tool in surgical applications other than standard MIVS.

\section{Drainage of Extensive Suprachoroidal Haemorrhages}

The current standard of care in the surgical management of extensive suprachoroidal haemorrhages is the drainage of the blood through posterior sclerotomies, either alone, or, more often, in combination with MIVS for removal of concomitant vitreous haemorrhage, retained lens material or retinal detachment surgery. ${ }^{7}$ Rezende et al. ${ }^{8}$ described the transconjunctival drainage of haemorrhagic choroidal detachments using 20-gauge vitrectomy trocars. We have good experience with an adjustment of this technique, using 23-gauge microincision vitrectomy trocars. The surgery should be performed after ultrasound confirmation of liquefaction of the choroidal haematoma. With the widespread use of valved trocars in MIVS, non-valved devices are less commonly available. However, the valve system can be easily opened with the cutting vitrector tip (see Figure 1). An intraocular infusion channel can be placed in the anterior chamber and a 23-gauge trocar is introduced transconjunctively in the most affected quadrant, approximately $7 \mathrm{~mm}$ 


\section{Figure 1: Opening a Valve System}

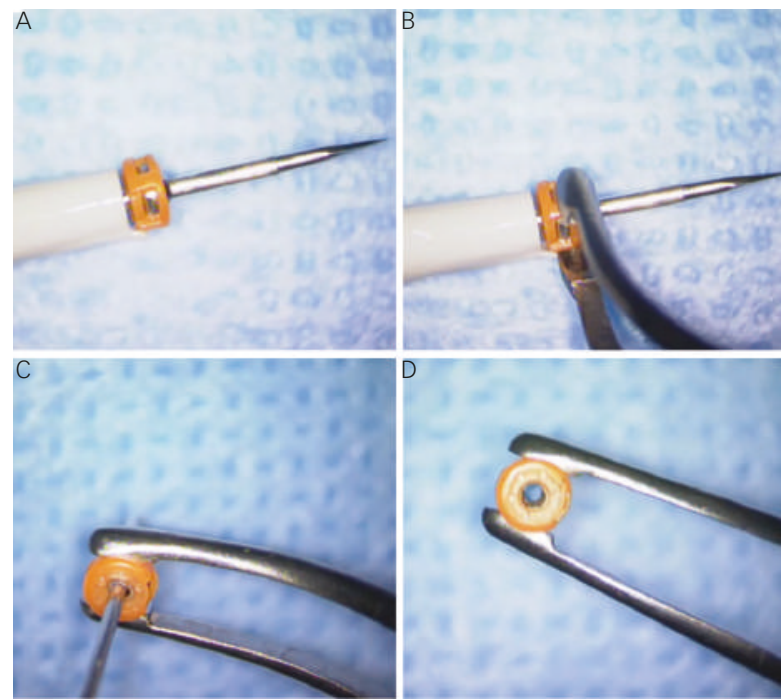

The valve can be easily removed from the trocar with the cutting vitrector tip (C).

Figure 2: Suprachoroidal Haemorrhage Drainage
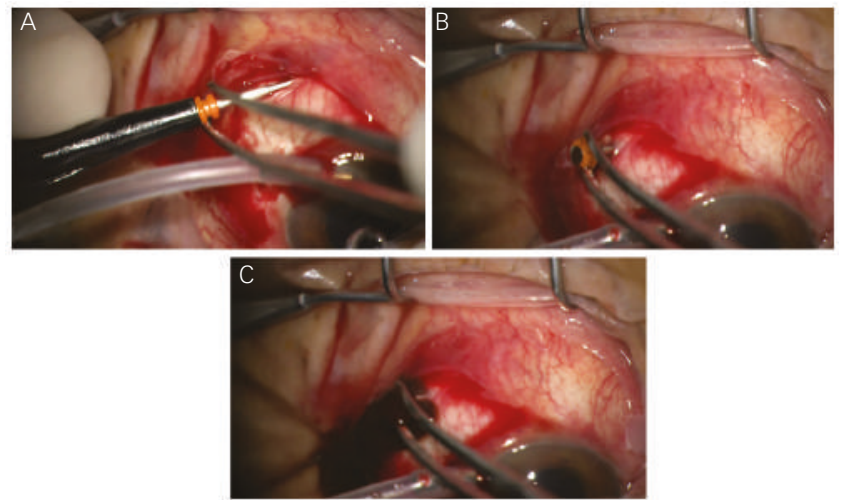

(A) The trocar is inserted with an oblique trajectory trough the conjunctiva and sclera, approximately $7 \mathrm{~mm}$ from the limbus. (B) The cutting edge is removed leaving the cannula in place. (C) The suprachoroidal haemorrhage is then drained.

\section{Figure 3: Trocar-assisted Pupilloplasty}

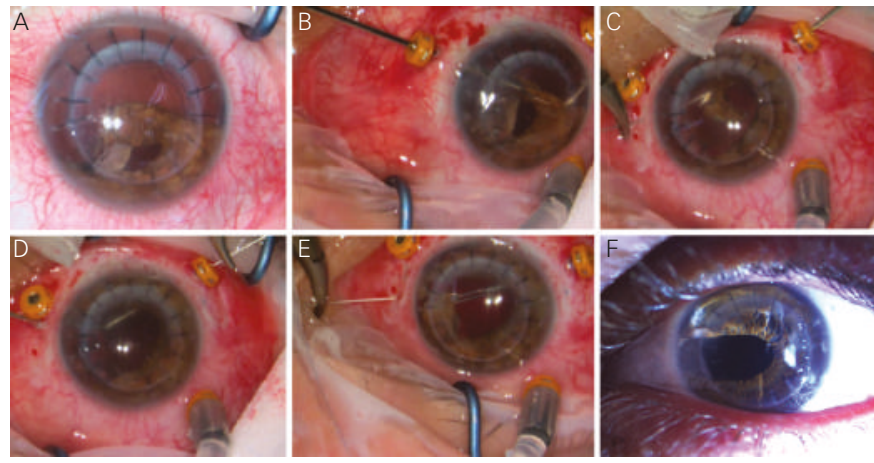

In a patient with a penetrating keratoplasty who suffered a traumatic lens luxation and iridodialysis. (A) Pre-operatively the iridodyalisis is clearly seen. (B) 23-gauge retinal forceps and a 25-gauge needle to suture the iris root. (C, D and E) A 10/0 prolene suture is passed and docked in the 25-gauge needle lumen at the port. These procedures are repeated to achieve the best possible result. (F) Slit-lamp photography of the patient, 3 months post-operatively.

from the limbus. The trocar should be inserted gently, in a 20 to $30^{\circ}$ angulation. After entering the cutting tip, only the cannula progresses, thus avoiding iatrogenic retinal damage. After removing the trocar
Figure 4: Trocar-assisted Pupilloplasty with Limbus Placement of the Trocar
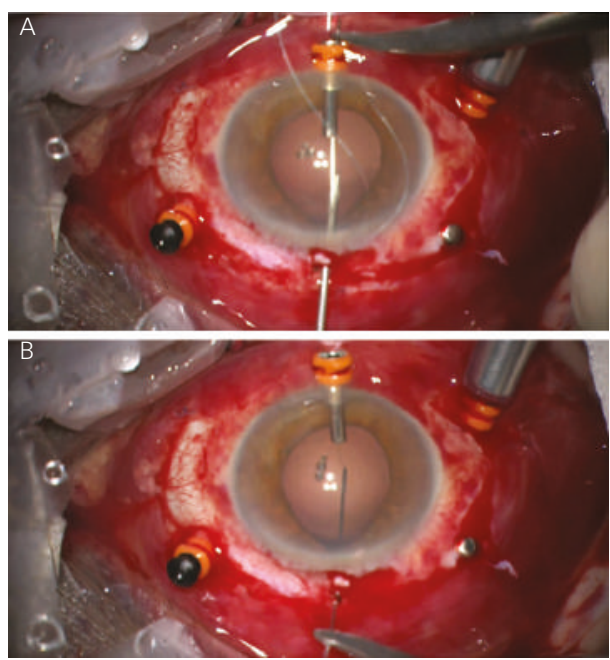

\section{Figure 5: Schematic Representation of Transconjunctival Trocar Placement}

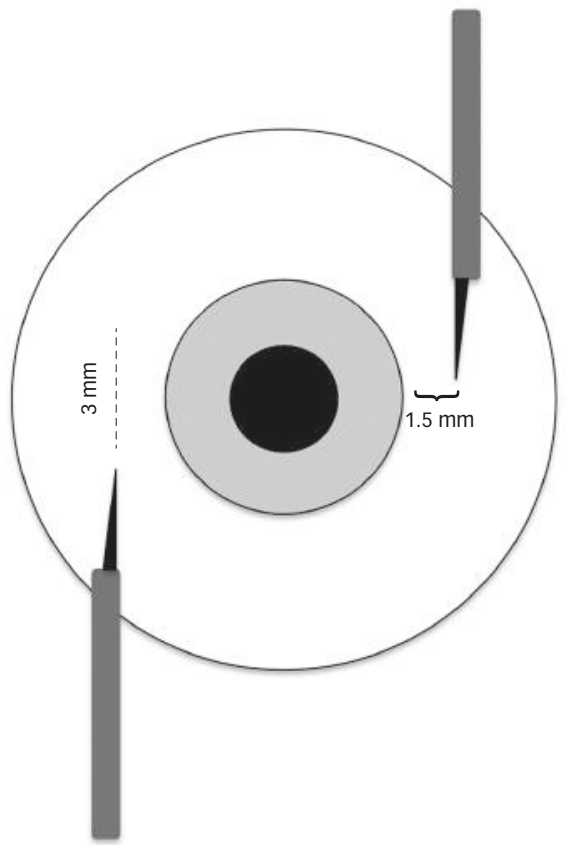

Two diametrically opposed $3 \mathrm{~mm}$ long scleral tunnels are prepared with 25-gauge trocars, $1.5 \mathrm{~mm}$ from and parallel to the limbus. Figure adapted from Totan and Karadag, 2012.10

blade, the cannula is repositioned in the suprachoroidal space to allow for the drainage of the blood (see Figure 2). Other openings may be subsequently performed to obtain the best possible drainage of the blood, with sutureless closing. Subsequent MIVS may follow, whenever necessary.

\section{Pupilloplasty}

Another conceivable use for microincision vitrectomy trocars is in complex anterior segment surgeries requiring pupilloplasty. The trocars put in place for pars plana vitrectomy can be used to facilitate iris sutures (see Figure 3). Alternatively, trocar placement in the limbus (see Figure 4) can also be used to pass sutures with or without docking, thus ensuring a free and easy passage of suture material through a 


\section{Vitreoretina}

\section{Figure 6: Trocar-assisted Intraocular Lens Fixation}
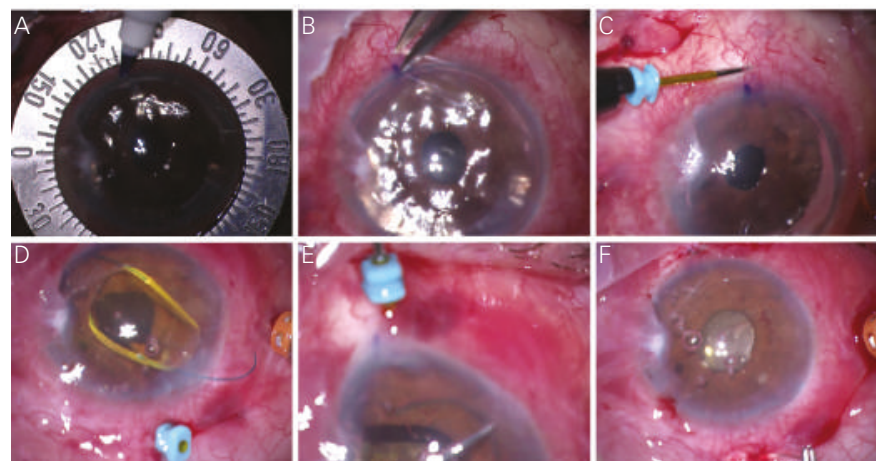

Two diametrically opposed secleral tunnels $1.5 \mathrm{~mm}$ from the limbus are performed using 25-gauge trocars (A, B and C). The foldable three-piece intraocular lens (IOL) is inserted in the anterior chamber (D) and then placed in the sulcus. The haptics are manipulated with the aid of 25- and 23-gauge retinal forceps so that they exit the eye through the port (E). Both haptics are then carefully placed in the scleral tunnels and the IOL is left in place (F).

small incision opening. At the end, the trocar is removed and often no suture of the limbic entrance is required. In our experience, the use of microincision vitrectomy trocars as coadjuvants of pupilloplasty surgery has proved both practical and effective, hence facilitating an intrinsically challenging surgical procedure.

\section{Trocar-assisted Intraocular Lens Fixation}

In patients with insufficient posterior capsule support after cataract surgery, a three-piece foldable intraocular lens (IOL) can be placed in the sulcus with a sutureless scleral fixation technique." Two diametrically opposed scleral tunnels are prepared with 25-gauge trocars, $1.5 \mathrm{~mm}$ from and parallel to the limbus (see Figure 5). A standard three-piece IOL is injected into the anterior chamber and the haptics are manipulated with the assistance of 25- and 23-gauge retinal forceps, through the paracentesis and the pars plana ports (see Figure 6). The haptics are then incarcerated in scleral tunnels and left in place. ${ }^{10}$ This technique is less time-consuming and provides less manipulation than the conventional scleral-suturing options.

\section{Conclusion}

Significant improvements in the design of microincision vitrectomy trocars over the last decade have significantly contributed to improve the success of MIVS. Moreover, these instruments have unveiled a new world of possibilities in ocular surgery, redefining surgical practices through limitless applications.
Thompson JT, Advantages and limitations of small gauge vitrectomy, Surv Ophthalmol, 2011:56:162-72.

2. Recchia FM, Scott IU, Brown GC, et al., Small-gauge pars plana vitrectomy: a report by the American Academy of Ophthalmology, Ophthalmology, 2010;117:1851-7.

3. Oshima Y, Wakabayashi T, Sato T, et al., A 27-gauge instrument system for transconjunctival sutureless microincision vitrectomy surgery, Ophthalmology, 2010;117:93-102 e2.

4. Fujji GY, De Juan E, Jr, Humayun MS, et al., A new 25-gauge instrument system for transconiunctival sutureless vitrectomy surgery, Ophthalmology, 2002:109:1807-12; vitrectomy surg
discussion 13.

5. Eckardt C, Transconjunctival sutureless 23-gauge vitrectomy, Retina, 2005;25:208-11.

6. Kadonosono K, Yamakawa T, Uchio E, et al., Comparison of visual function after epiretinal membrane removal by 20-gauge and 25-gauge vitrectomy, Am J Ophthalmol, 2006;142:513-5.

7. Chu TG, Green RL, Suprachoroidal hemorrhage
Surv Ophthalmol 1999:43:471-86.

8. Rezende FA, Kickinger MC, Li G, et al. Transconjunctival drainage of serous and hemorrhagic choroidal detachment Retina, 2012;32:242-9.

9. Gabor SG, Pavlidis MM, Sutureless intrascleral posterior chamber intraocular lens fixation, I Cataract Refract Surg, 2007;33:1851-4

10. Totan $Y$, Karadag R, Trocar-assisted sutureless intrasclera posterior chamber foldable intra-ocular lens fixation, Eye, 2012;26:788-91. 